\title{
Protée
}

\section{L’atlas de la vie après la mort}

\section{Richard Purdy}

Volume 27, numéro 3, 1999

L'imaginaire de la fin

URI : https://id.erudit.org/iderudit/030572ar

DOI : https://doi.org/10.7202/030572ar

Aller au sommaire du numéro

Éditeur(s)

Département des arts et lettres - Université du Québec à Chicoutimi

\section{ISSN}

0300-3523 (imprimé)

1708-2307 (numérique)

Découvrir la revue

Citer cet article

Purdy, R. (1999). L'atlas de la vie après la mort. Protée, 27(3), 70-82.

https://doi.org/10.7202/030572ar d'utilisation que vous pouvez consulter en ligne.

https://apropos.erudit.org/fr/usagers/politique-dutilisation/ 


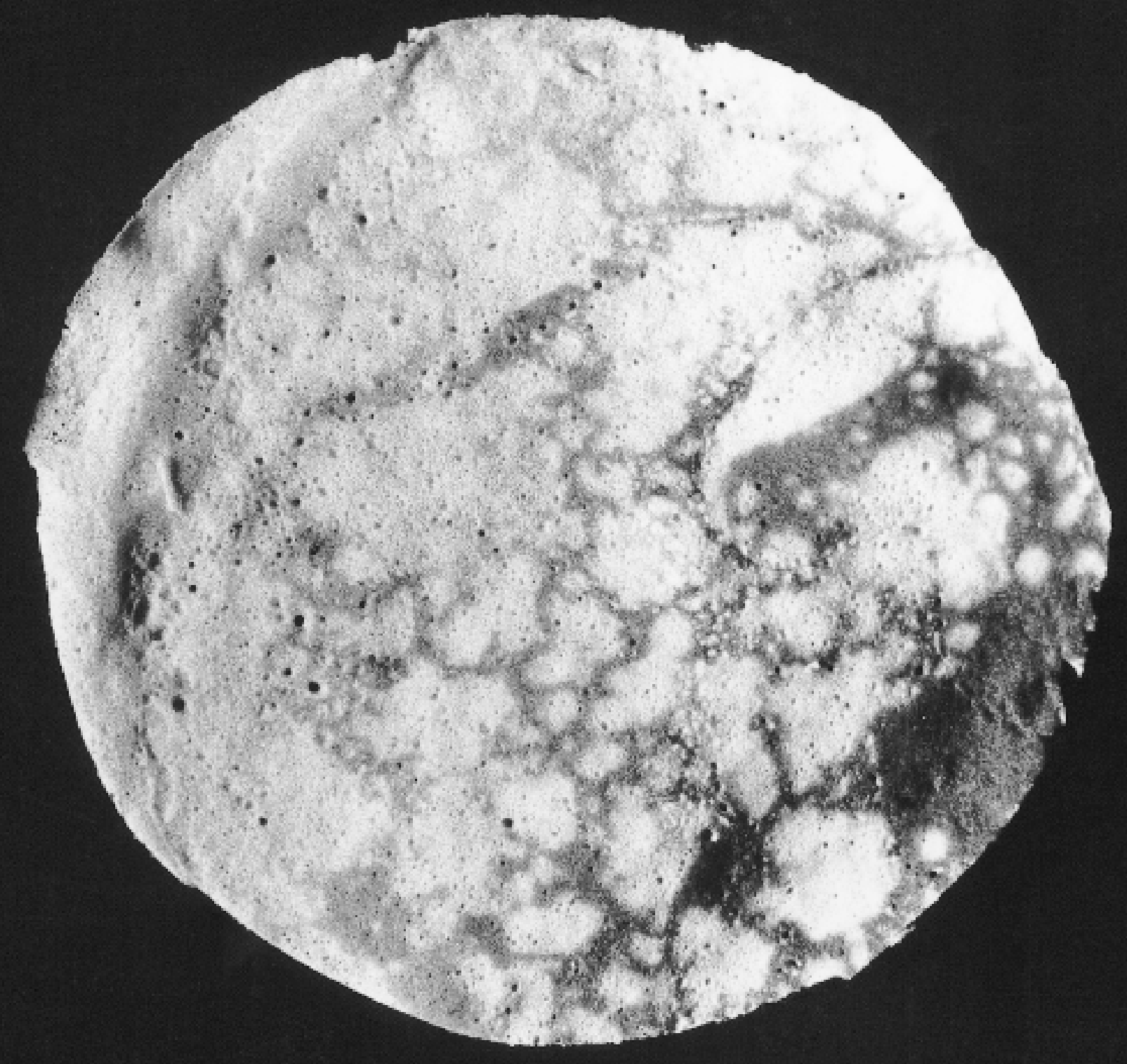

Areoarcheologic: Archéologie sur planète M ars. Installation : échafaudages, aquariums, brique concassée, systèmes électriques. Galerie Koffler, Toronto (O ntario), 1997. Image: Crêpes de $23 \mathrm{~cm}$ de diamètre. Photo de l'artiste. 


\section{L'ATLAS DE LA VIE APRÈS LA MORT \\ Richard Purdy \\ Traduction de Bertrand Gervais}

II vaut mieux un monde sans pain qu'un monde sans Dieu, comme un monde inhabité est préférable à un monde profane.

Saint Antoine de Padoue

LU CIFER déjoue toute pensée humaine. II est le Prince du mensonge. Son histoire est le sujet de cet opéra.

Il est d'abord connu comme un Être de lumière (- tous les anges sont des Êtres de lumière),

Peu importe sa stature (- il était le plus grand de tous les anges),

O u les raisons de sa chute (- le matérialisme scientifique),

Il chancelait,

Et il tomba...

c'est-à-dire qu'il a pris du poids ${ }^{1}$.

Au début de sa chute, LU CIFER était une lumière éteinte (- soit un ensemble d'atomes instables formés d'un nuage d'électrons libres)

Dans cet état, il continua sa chute, à travers les champs gravitationnels de nombreuses galaxies, à travers les quadrants de la Voie Lactée, dans l'orbite du système solaire, jusqu'à la troisième planète, pour atteindre finalement l'atmosphère de la Terre ${ }^{2}$.

Au moment de son entrée dans l'atmosphère de la Terre, le corps de LU CIFER se transforma encore en une matière plus lourde que l'air qu'il traversait. S'il ne l'avait fait, sa chute aurait été stoppée.

Aussi, à cet instant, il devint humidité ${ }^{3}$.

1. La structure photonique de la lumière est constituée à la fois de particules et d'ondes, bien qu'aucun état ne puisse être mesuré avec précision. Pour que la lumière devienne impure, elle ne doit plus être de la lumière.

2. Cette partie de la chute, bien qu'à peine esquissée dans notre récit, est la plus longue partie de la chute de LU CIFER, et sans doute la plus mouvementée.

3. L'humidité, cependant, était polluée. LU CIFER est une matière malsaine: inutile, empoisonnée, toxique

- rejetée dans l'atmosphère dans laquelle on la trouve. 


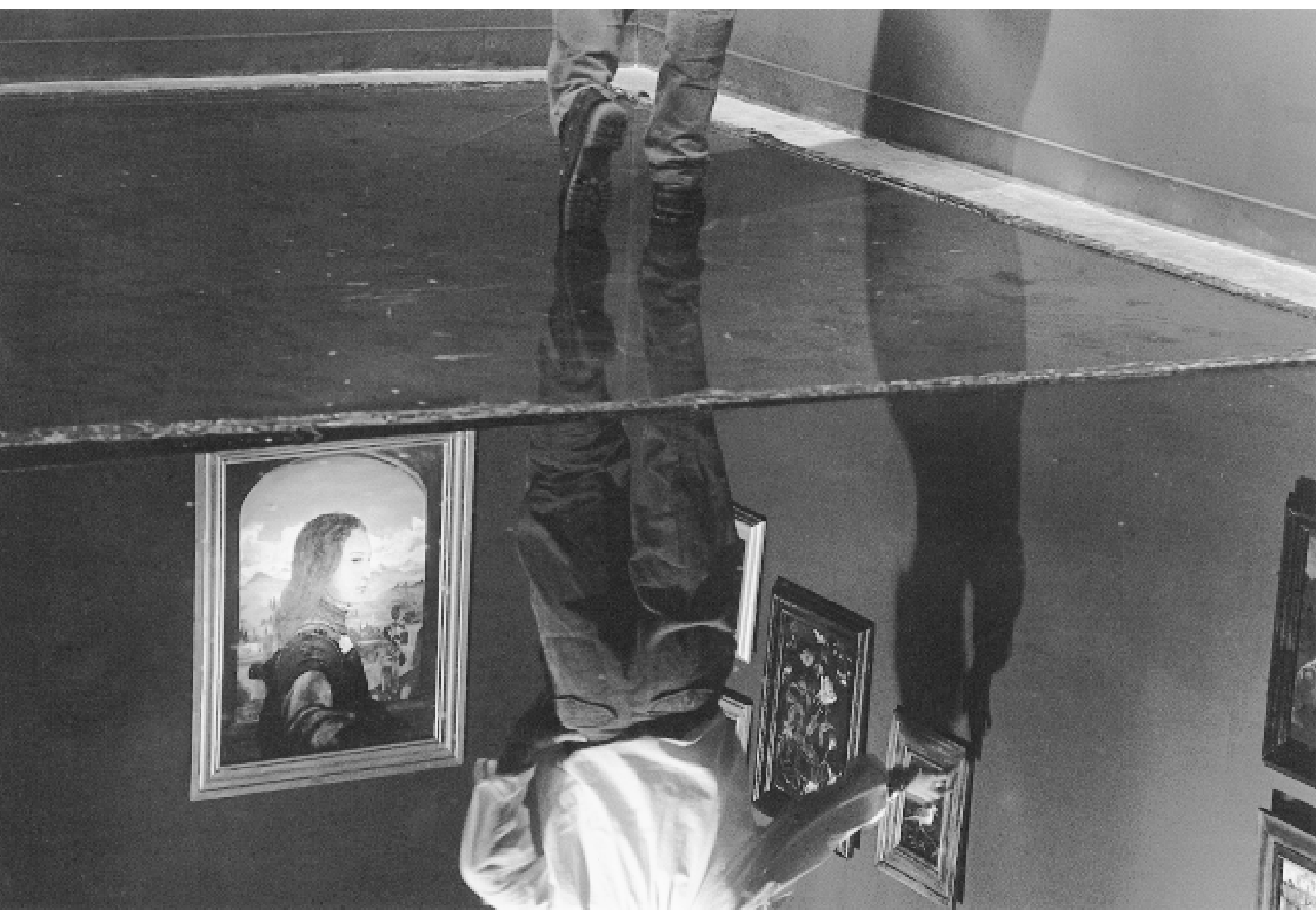

La Caduta di Lucifero. Installation à la Galerie Christiane Chassay (M ontréal) et au M ercer U nion (Toronto, O ntario) ; 65 m 2, 1995. Photo : Juan Felipe Argaez. 


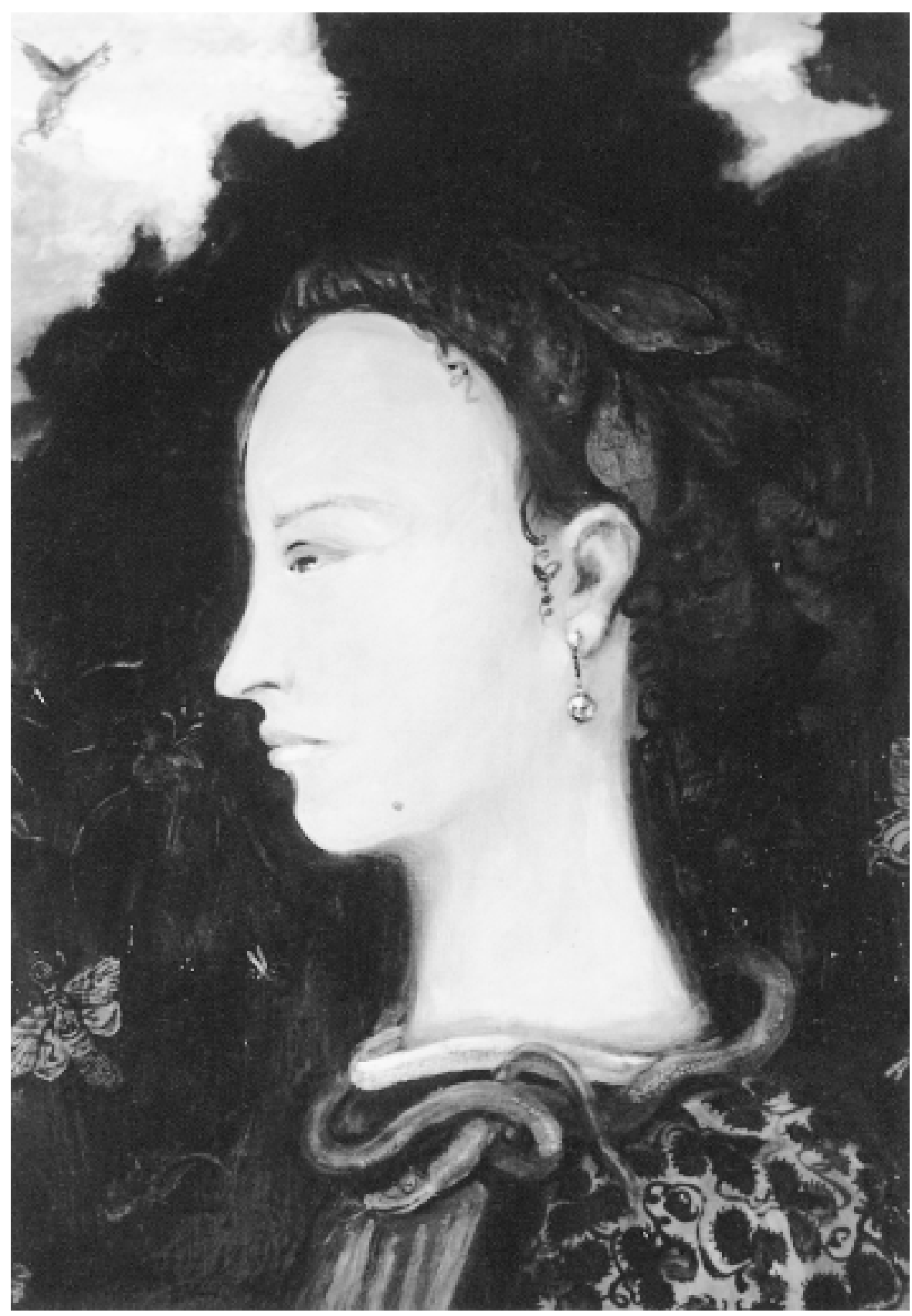

La Caduta di Lucifero: «Andromaque». Peinture à l'huile sur toile, 2 × 3,05m. 1995. Collection du Musée du Q uébec. 


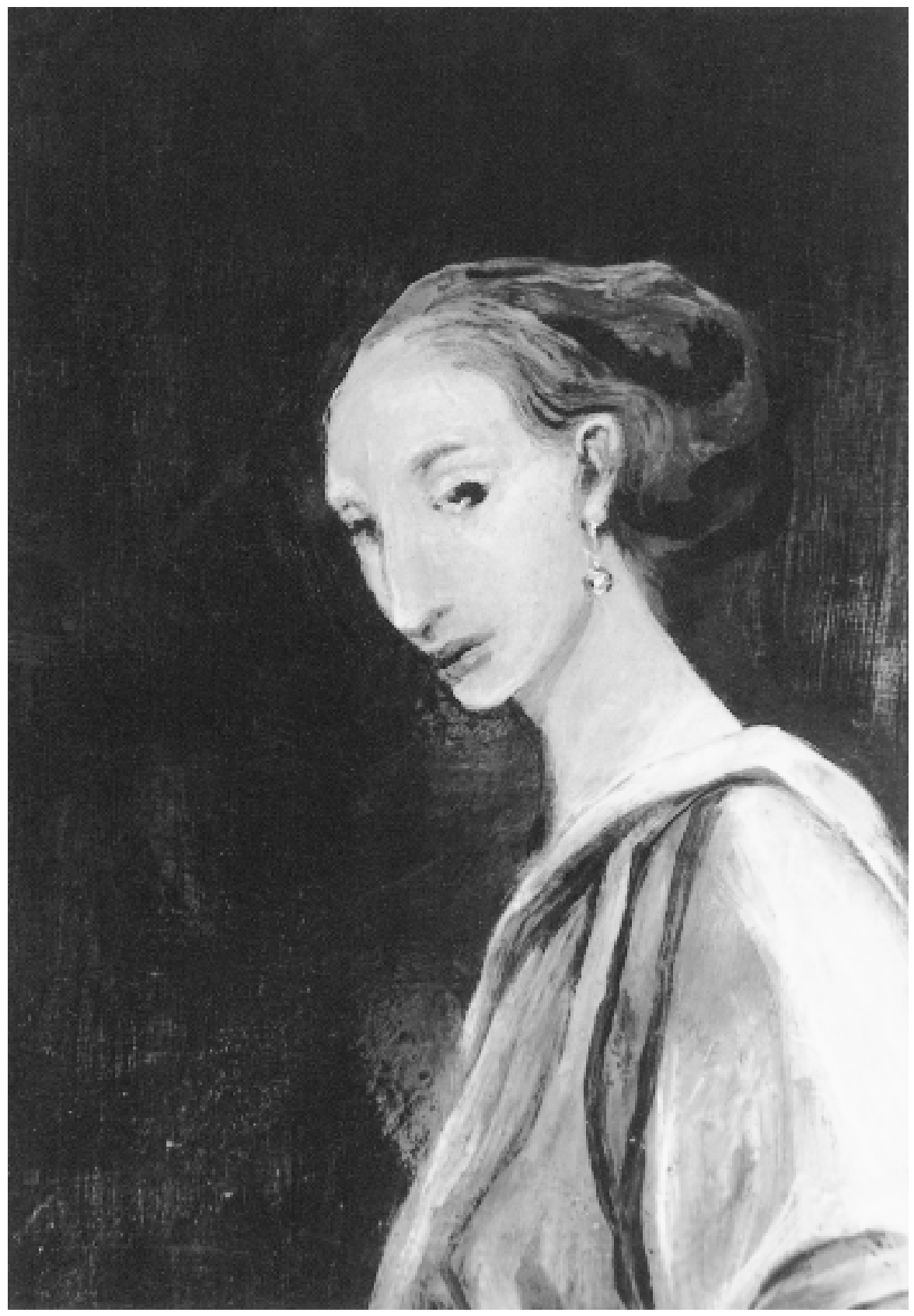

La Caduta di Lucifero: «Laurie Walker ». Peinture à I'huile sur toile, 110 × 80 cm, 1994. 
La chute fut ralentie par la présence de nuages au-dessus de la terre. À nouveau, le corps de LU CIFER changea d'état, et tomba...

Et il se transforma en une matière plus lourde que la vapeur - une eau dilatée ${ }^{4}$.

L'eau pestilentielle se mêla à la pluie atmosphérique et, à cet instant, la traversa sous la forme de cendres ${ }^{5}$. La chute des cendres fut ralentie par la présence de vents à la surface de la terre. La chute de LU CIFER ne se fit pas en ligne droite...

elle se tordit et tourna, et quelquefois encore remonta. Mais son inexorable direction était vers le bas - parce qu'elle est, après tout, une chute ${ }^{6}$.

À ce moment, LU CIFER devint un être constitué d'une terre stérile et surexploitée ${ }^{7}$.

La terre, la couleur d'une anti-couleur sans couleur (antirouge, antibleue, antiverte), tomba du ciel. Le Prince SATAN

rejoignit le monde des hommes. Comme il heurtait la masse de I'humanité, il se

4. II est à noter que, jusqu'à présent dans notre récit, le corps de l'ange déchu se transforme toujours en une matière plus lourde que ce à travers quoi il passe.

5. Le gouvernement a causé tout un émoi dans la population en transformant tout son papier-monnaie en pièces métalliques. Les riches se sont plaints du poids de la nouvelle monnaie et du fait qu'elle était sale d'avoir été touchée par toutes les strates de la population. Les pauvres ont protesté que tout leur argent avait été transformé en menue monnaie. Pour toute réponse, le gouvernement a mis sur pied un programme pour laver l'argent régulièrement, un sou à la fois, et pour distribuer l'eau du lavage aux pauvres afin qu'ils la boivent.

6. Un jour, un homme demanda à sa femme, pendant qu'ils faisaient l'amour sur le sol, si elle avait remonté I'horloge ce matin-là. La femme eut peur que cette question inappropriée rende l'enfant qui pouvait naître incapable, tout au long de sa vie, de suivre le fil d'une pensée.

7. À la mort de son mari, une musicienne avait retiré les cordes de sa harpe et l'avait placée dans le grenier, le temps de son deuil. Après de longues et tristes années, elle a enfin enlevé ses vêtements noirs, elle les a pliés et rangés. Grimpant l'escalier du grenier pour récupérer son instrument de musique, elle a découvert que sa harpe avait été enserrée de fils d'araignée et que les lombrics et les araignées en avaient joué allègrement tout au long de son deuil. 


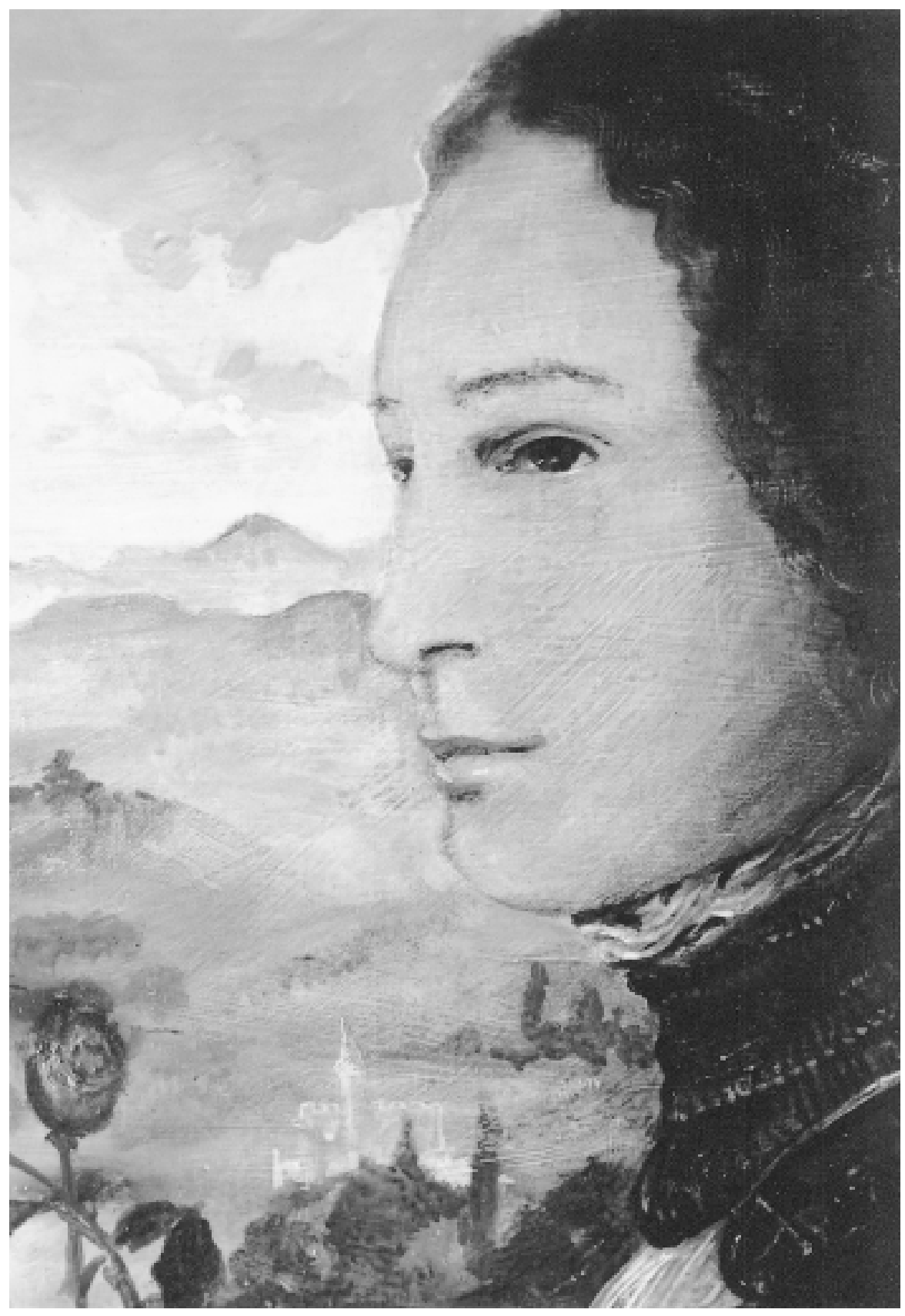

La Caduta di Lucifero: «Homme». Peinture à l'huile sur toile, 184 x 120cm, 1994. Photo de l'artiste. 
transforma à nouveau, accéléra sa chute et devint la merde des sous-alimentés ${ }^{8}$.

Le corps de LU CIFER, après avoir traversé de part en part l'humanité et avoir été chié sur la surface de la planète, se modifia en une matière plus lourde que la terre, continuant sa chute. Le corps jadis angélique pénétra l'humus et se métamorphosa en lombrics ${ }^{9}$.

Elle fut brève la chute du prince des TÉN ÈBRES dans les profondeurs. Et le corps de l'impie se changea en roc et s'enfonça profondément au sein de la terre. II ne devint pas une pierre utile, du marbre, de la calcédoine ou du calcaire - mais une roche inutile, fissile, prête à s'émietter ${ }^{10}$.

Le roc frappa le roc et ralentit le mouvement de chute de ce corpus corruptilis. Mais la chute de LU CIFER ne révoquait pas les processus naturels tels que nous les connaissons. U ne roche prendrait plusieurs siècles pour traverser le corps de la terre (et nous pouvons lier ce fait au processus de stratification). Pour LU CIFER et DIEU, cependant, ce passage eut lieu en une fraction de seconde. Et pendant qu'il poursuivait sa chute, le corps du démon se changea inexorablement en une des matières les plus empoisonnées...

8. Le cadavre qui a été brûlé dans le lieu sacré est un faux. La parenté, terrifiée par la certitude d'être hantée par l'esprit de leur parent diabolique et impie, avait volé le corps d'une personne sainte et l'avait substitué au corps de leur frère corrompu! M ais, au dernier instant, ils se trompèrent en identifiant les corps. L'homme diabolique fut brûlé (ses cendres montèrent au ciel), pendant que l'homme bon et saint se décomposait dans la rivière toute proche, mangé par la vermine et les chiens.

9. La rivière Masji-masid coule simultanément vers l'est et l'ouest à partir du centre de la ville du même nom. Personne ne peut expliquer le prodige, mais cela fait de la région un important centre de commerce, parce qu'elle peut approvisionner deux fois plus de ports que toute autre ville. Un cadavre jeté dans le port de Masji-masid ne disparaît jamais, car les remous de l'eau du port le font tourner en rond sans fin.

10. On retrouve encore aujourd'hui d'étranges roches à l'endroit où le corps de LU CIFER a pénétré la terre (quelque part en Irlande du Nord). Ces roches sont d'un noir de jais, mais très légères. Elles peuvent, étonnamment, s'embraser au contact d'une flamme et émettre une chaleur intense. 


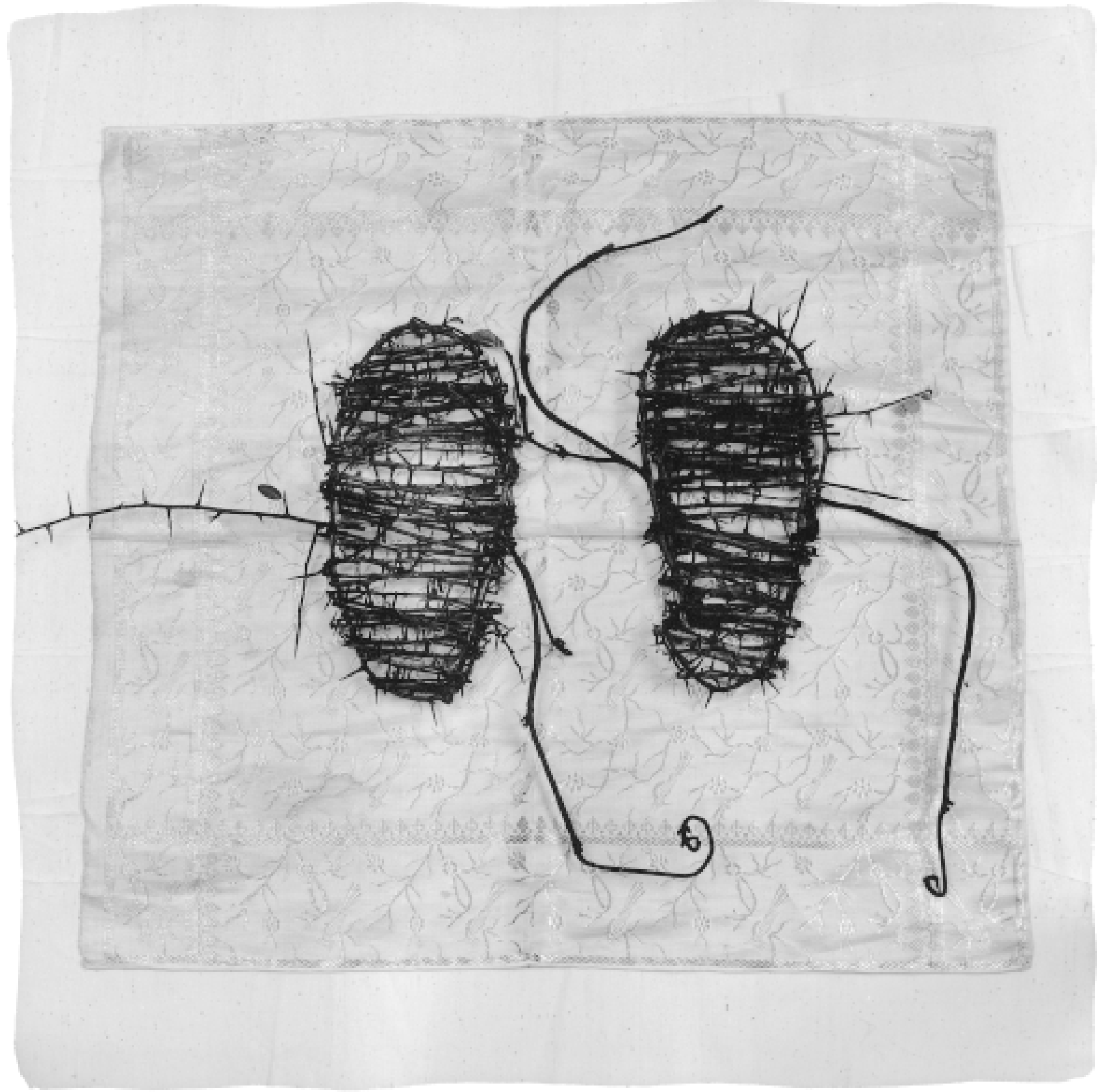

Sandales d'épines. Patiner sur l'œil. CIAS Crestet (France). Broderie sur soie, matière végétale, $20 \times 24$ x 10cm, 1993. Photo : François Blanquie. 
le plomb: le domestique du diable ${ }^{11}$.

Au fur et à mesure que le corps satanique s'abîme plus profondément dans les entrailles de la planète, sa constitution nous échappe davantage. La terre est opaque à la vision humaine. N ous savons, cependant, que l'impie a continué sa chute. Et s'il a chu, alors son corps est devenu toujours plus lourd que le matériau à travers lequel il passait. Sinon comment pourrait-il continuer à descendre? Sa route serait interrompue. De par la constitution du plomb, nous pouvons présumer que le prochain état du corps de LU CIFER doit correspondre à la douleur ${ }^{12}$.

La douleur dans sa condition matérielle a continué sa descente, jusqu'à rejoindre le bord du noyau de la terre. LU CIFER a revendiqué le noyau magmatique de la terre comme Enfer, mais il est passé rapidement au travers de son domaine. Invisible, et impossible à connaître pour tout être vivant, nous devons émettre I'hypothèse que le corps de SATAN est devenu une masse d'intelligence incorporelle, l'origine de tout mal ${ }^{13}$.

À partir d'ici, nous avons, et ne pouvons avoir, une idée précise du destin de LU CIFER, sauf à dire qu'il est devenu une forme de matière extrêmement dense et, en même temps, un principe du mal surpassant toute intelligence. II a traversé le spectre de notre connaissance...

11. C'est un fait avéré que la réputation de l'instabilité mentale des artistes est basée sur plus que des stéréotypes ou une malice professionnelle. Les recherches ont montré les effets néfastes du plomb sur le cerveau humain; il provoque des hallucinations, des illusions et des visions paranoïaques. Les artistes sont reconnus pour avoir fait, depuis toujours, un usage assidu du plomb dans leurs préparations de peinture.

12. Dans la vallée de la Loire, un groupe de moines cénobites vénérait le nom d'O rtémis, qui avait été un des soldats romains présents après la crucifixion de Jésus-Christ. Après le dernier cri d'agonie du Christ, sa tête tomba sur sa poitrine et la couronne d'épines roula par terre, aux pieds d'O rtémis. Instantanément converti au christianisme, O rtémis détacha sa scandale droite et glissa son pied dans la couronne épineuse - qui lui mordit immédiatement la chair. II se rendit ainsi chaussé jusqu'en Turquie, où il mourut au bout de son sang. Les membres de l'ordre d'O rtémis portent encore de nos jours des scandales d'épines en mémoire de l'exceptionnelle pénitence de saint O rtémis. 13. Pendant plus de soixante ans, les historiens ont daté les sites médiévaux à partir des traces de fers à cheval présentes sur le sol stratifié. Les fers à cheval médiévaux étaient toujours marqués du sceau de leur fabricant, ce qui en facilitait la datation. Récemment, il a été découvert cependant que les vers de terre, lorsqu'ils défèquent, sont attirés par les objets métalliques, recouvrant le fer à cheval ordinaire avec plus d'un centimètre de matières fécales par année. Comme la merde des vers de terre est deux fois plus lourde que la terre, cette production des vers annalides peut pousser le fer à cheval plus profondément dans les couches du sol qu'il ne devrait l'être - bouleversant ainsi les dates attribuées aux stratifications. Cette découverte biologique a ébranlé le système de datation de l'histoire médiévale, maintenant considéré comme fallacieux. 


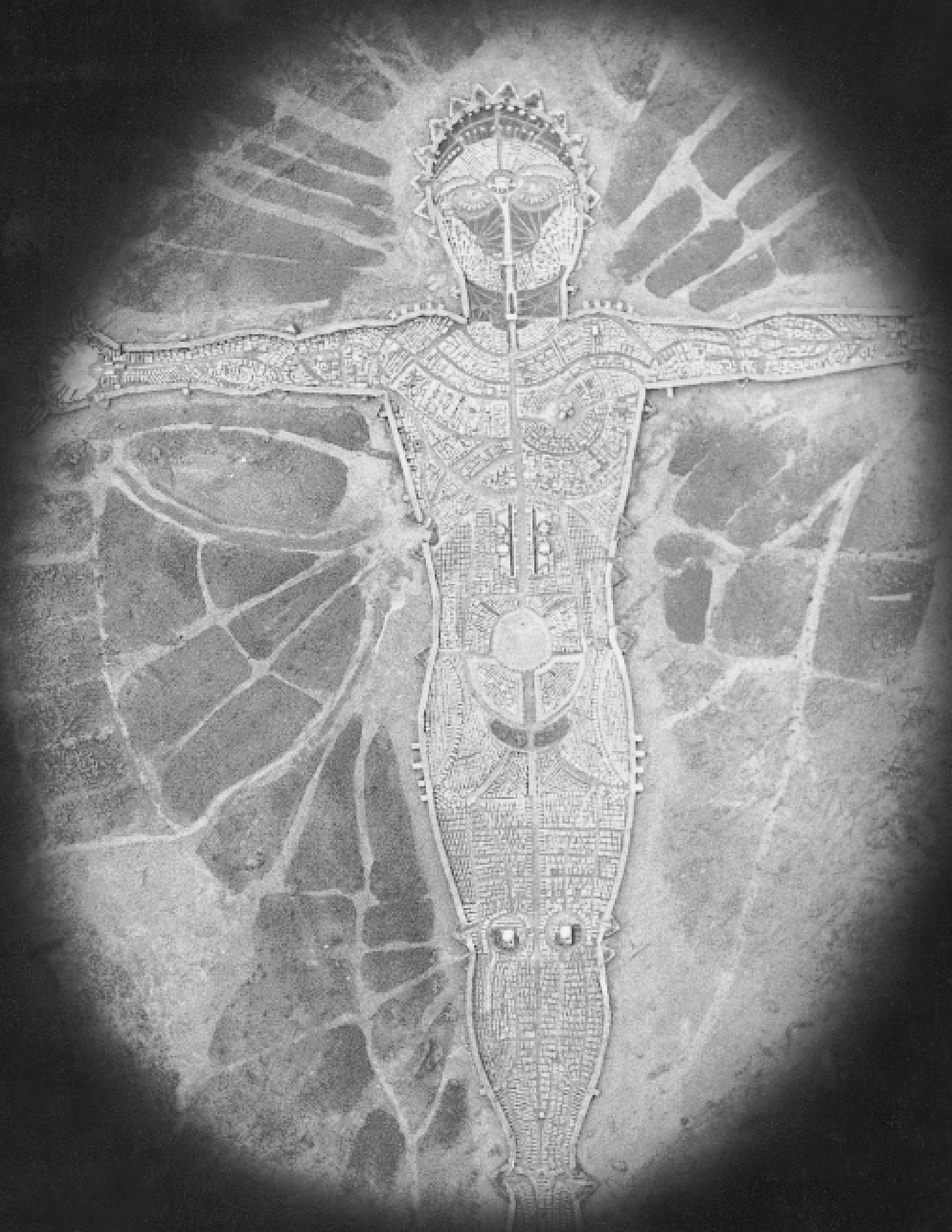

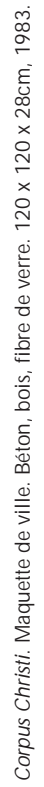


Et il s'en est échappé par le fond ${ }^{14}$.

14.

Ich fiel, es ist wahr, doch

noch im Fall

Verlor ich M ut und Stärke

nicht.

Ich fiel, ich fiel, ich fiel,

nein, nein, ich varlor sie

nicht!

Whol war, als aus dem

himmelszeit er mich

vertrieb, der herr mir

überlegen;

doch Mensch geworden,

ist er meiner Wut,

Indem er sterben muste,

unterlegen.

Die Tiefen will ich

durcheinanderrüttein,

Von ihrem Mitteipunkt

will ich die Erde

ershüttern und in alle Welt

zerstreuen: mit meinem Atem

in die lüfte

mit meinen Seufzer in das

feuer,

und mein Verlangen trägt den

Krieg zum Himmel

Und mit unheimlichem

Dunkel die gedanken aus dem

schwachen

Geist der Menschheit ganz

verstören.
J'ai chu, il est vrai, mais

dans ma chute

je n'ai perdu ni force ni

ardeur

J'ai chu, J'ai chu, J'ai

chu,

non, non, je n'ai rien perdu!

Si Dieu, quand il m'a

banni des sphères,

était alors le plus fort,

homme maintenant, en

mourant,

il a succombé à ma

fureur.

Je bouleverserai les

abîmes j'arracherai la

terre de son axe

et I'éparpillerai

je porterai la guerre

à l'air d'un souffle,

au feu d'un soupir, au ciel

d'une haleine!

Et avec de nuisibles

ténèbres

je confrondrai toutes les idées de l'infirme pensée humaine.

no, no, I lost not strength!
And with noxious darkness

confound all processes

of feeble human thought.

I fell, 'tis true, but in

falling I lost neither

strength nor courage,

I fell, I fell, I fell

Though when he threw

me through the spheres

God was then the

stronger, now as a man,

He has

succumbed

to my hate by dying.

I'Il throw Hell into confusion

I'Il convulse the Earth

from within

and scatter it abroad

into the air with my

breath

and into the fire with my

sighs,

and storm the gates of

Heaven with ambition!

And with noxious
darkness
confound all processes
of feeble human thought.

E con tenebre nocenti

delle inferme umane

menti

O gn'idea confonderò.

Caddi, è ver, ma nel cadere $\mathrm{N}$ on perdei forza né ardire,

Caddi, Caddi, Caddi

no, no, non perdei!

Per scacciarmi dalle sfere Se più forte allor fu Dio, Or fait 'uomo, al furor moi

Per ceduto ha con morire.

Sconvolgerò gl'abissi

Dal uo centre commossa Dissiperò la terra,

All'aria coi respiri,

Al fuoco coi sospiri

Con gli aneliti al Ciel

muoverò guerra!

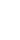

.




\section{LE MU SÉE DIABO LIQ U E DE RICHARD PURDY}

In mplacable est la chute de Lucifer à travers les strates de l'univers. Impitoyable sa descente sous la ligne du texte.

L'Atlas de la vie après la mort est une traversée. Et sa trajectoire croise des œuvres diverses de l'artiste. La principale est La Caduta di Lucifero, I'installation montée à la Galerie Christiane Chassay à M ontréal, à la G alerie O bscure et au Mercer U nion à Toronto. De nombreuses photographies en témoignent.

Dans ces salles, sont accrochées sur les murs noirs vingt toiles, réunies en groupes compacts, dans une configuration digne des «beaux-arts». Des paysages, des natures mortes, des portraits, peints dans le style des académies du 19 e siècle. Mais, par une technique de dé-restauration, les surfaces de ces toiles ont été abîmées et dégradées par l'artiste, pour les rendre plus abstraites, leur donner cette qualité des peintures impressionnistes.

Détail inusité, ces toiles dérestaurées ( «unrestored») ont toutes été suspendues la tête en bas. Elles sont en état de chute. Renversées sûrement comme doit l'être Lucifer dans son monde des ténèbres. Le sol des galeries, quant à lui, est recouvert d'une nappe d'eau d'un centimètre de profond, que contient un géotextile noir. Q uand l'eau est calme et que rien ne vient perturber sa surface, elle se transforme en une surface de réflexion, où réapparaissent à l'endroit les tableaux des murs. Un quai de bois est déposé sur cette nappe afin d'amener les visiteurs au centre des salles. De là, ils peuvent contempler les réflexions, ces tableaux aux perspectives corrigées par les ondes lumineuses, ou encore mettre les pieds dans l'eau et s'approcher des murs, marchant parfois directement dans certains tableaux aux dimensions importantes (jusqu'à $3,66 \mathrm{~m}$ ), mais brouillant à coup sûr les réflexions par leurs pas. Rien n'est plus fragile que l'eau. Q ue cette surface dont le calme au moindre heurt se rompt.

À cette série de photographies, s'ajoutent quelques pièces tirées du répertoire de l'artiste, qui répondent aux besoins de L'Atlas de la vie après la mort, à cette production artistique où convergent textes et œuvres photographiées, fictions archéologiques, scientifiques et historiques, et œuvres multimédiatiques. Ainsi, l'étrange planète tirée de la série de l'Aréoarchéologie, la vue aérienne de Corpus Christi, s'ils viennent scander les étapes de la chute de Lucifer, témoignent aussi, de façon absolue, d'un esprit sans cesse déporté aux limites de l'imaginaire. 